\title{
Softening Treatment of Silk/Poly(Lactic Acid) Blend Fabric
}

\author{
Kaiqiang Liu ${ }^{\mathrm{a}}$, Jin-Ping Guan ${ }^{\mathrm{b}}$ and Ren-Cheng Tang ${ }^{\mathrm{c}}$ \\ National Engineering Laboratory for Modern Silk, College of Textile and Clothing Engineering, \\ Soochow University, 199 Renai Road, Suzhou 215123, China \\ a kliu@suda.edu.cn, ${ }^{\mathrm{b}}$ guanjinping@suda.edu.cn, ${ }^{\mathrm{c}}$ tangrencheng@suda.edu.cn
}

Keywords: Poly(lactic acid); Silk; Blend; Softener; Hydrophilicity.

Abstract. Silk/poly(lactic acid) (PLA) blend fabric is a new textile material, and it suffers from hardening in the process of wet and dry heat treatment. Softening treatment usually becomes a required process to compensate the loss of softness. In this work, the softeners having different chemical structures were applied to improve the handle of silk/PLA fabric, and the effects of softening treatment on the handle, hydrophilicity, color parameters and color fastness of silk/PLA fabric were discussed. It was found that the softener having polyether fatty acid ester component was able to impart good softness to the fabric, guarantee the inherent hydrophilicity of the fabric, and exert minimal impact on the color parameters and wash color fast of the dyed fabric.

\section{Introduction}

In recent years, bio-based poly(lactic acid) (PLA) fiber has drawn great attention as a promising replacement of petroleum-based poly(ethylene terephthalate) (PET) fiber [1], and it has been widely applied to develop new textile products. Silk/PLA blend fabric combines the outstanding characteristics of silk and PLA fibers such as softness, hygroscopicity, comfortableness, good biocompatibility and easy biodegradability [1, 2], and it has been a fashionable textile product. Before a greige silk/PLA blend fabric becomes a final product, it must be subjected to scouring, dyeing and mechanical and chemical finishing. Because PLA fiber has a low glass transition temperature, the silk/PLA fabric often suffers from hardening in the process of wet and dry heat treatment [3]. In this case, softening treatment becomes a required process in order to compensate the loss of softness. In addition, a nice, soft handle is often the decisive criterion for buying a textile [4]. Therefore, softening treatment has gained great importance in the finishing of silk/PLA fabric. In this work, the softeners having different chemical structures were applied to the finishing of silk/PLA fabric, and the effects of softening treatment on the handle, hydrophilicity, color parameters and color fastness of silk/PLA fabric were discussed. This research aimed to provide the basis for selecting the suitable softeners to improve the handle of silk/PLA fabric according to the requirements of final products.

\section{Experimental}

Materials. The scoured silk/PLA satin fabric was friendly provided by Jiangsu Xinmin Textile Science and Technology Co., Ltd.; its warp and weft were silk and PLA fiber, respectively, and the mass ratio of the two fibers was about 1:1. The commercially produced softeners were used, and their trade names, components and manufacturers are listed in Table 1. Disperse Red 3B (C.I. Disperse Red 60) and Dispertex RP-131 (a disperse agent) were commercial products. Acetic acid, sodium hydrosulfite and sodium bicarbonate were laboratory reagent grade chemicals.

Dyeing. The fabric was dyed in the XW-ZDR oscillated dyeing machine using the solution consisting of 2\%owf dye, 2g/L Dispertex RP-131 and 0.3 g/L acetic acid, and a 50: 1 liquor ratio. After immersing the fabric in the dye solutions at $40{ }^{\circ} \mathrm{C}$, the temperature was increased to $110{ }^{\circ} \mathrm{C}$ at 1 ${ }^{\circ} \mathrm{C} / \mathrm{min}$ and held for $50 \mathrm{~min}$, then cooled to $70{ }^{\circ} \mathrm{C}$ at $1{ }^{\circ} \mathrm{C} / \mathrm{min}$. At the end of dyeing, the dyed fabric was washed in the mixture solution of $2 \mathrm{~g} / \mathrm{L}$ sodium hydrosulfite and $1 \mathrm{~g} / \mathrm{L}$ sodium bicarbonate at 70 ${ }^{\circ} \mathrm{C}$ for $10 \mathrm{~min}$, and then rinsed thoroughly in distilled water, and allowed to dry in the open air. 
Table 1. Softeners used

\begin{tabular}{llll}
\hline Trade name & Component & Manufacturer & \\
\hline $\begin{array}{l}\text { Ablusoft } \\
\text { ALS-P }\end{array}$ & Amide ester & Taiwan Surfactant Corp. & \\
Aurigt CS-L & Cationic fatty imidazoline & $\begin{array}{l}\text { Suzhou Aurigt Chemical Co. Ltd., } \\
\text { China }\end{array}$ & $\begin{array}{l}\text { Hangzhou Xiasha Hengsheng } \\
\text { Chemical Co. Ltd., China }\end{array}$ \\
$\begin{array}{l}\text { RS-WR } \\
\text { Softener RS-C }\end{array}$ & Fatty acid amide & $\begin{array}{l}\text { Hangzhou Xiasha Hengsheng } \\
\text { Chemical Co. Ltd., China }\end{array}$ \\
Softener RS-M fatty acid ester & $\begin{array}{l}\text { Polyether fatty acid ester } \\
+ \text { a small amount of fatty acid }\end{array}$ & $\begin{array}{l}\text { Chemical Co. Ltd., China } \\
\text { Comide }\end{array}$ & \\
& & &
\end{tabular}

Softening. The fabric was first immersed in the solution of a softener $(10 \mathrm{~g} / \mathrm{L})$ for $5 \mathrm{~min}$, and then padded in two dips and nips using a two-roll laboratory padder with a wet pick-up of about $65 \%$. The padded fabric was dried at $100{ }^{\circ} \mathrm{C}$ for $3 \mathrm{~min}$.

Measurements. The capillary effect of the fabric (length $25 \mathrm{~cm}$; width $5 \mathrm{~cm}$ ) was evaluated by the vertical wicking test, and the capillary value was expressed by the rising height of distilled water along the fabric length orientation after the fabric bottom was immersed into water for $30 \mathrm{~min}$. The fabric hand or softness was subjectively evaluated by seven student participants: rating 1 and rating 10 represent the poorest and best hand, respectively; the average of seven evaluations was used. The CIE L*, C*, and h color coordinates (Lightness [ $\left.\mathrm{L}^{*}\right]$, chroma [C*], and hue $[\mathrm{h}]$ ) and the apparent color strength $(\mathrm{K} / \mathrm{S})$ of the dyed fabric were measured by the UltraScan PRO reflectance spectrophotometer (Hunter Associates Lab. Inc, USA) using illuminant D65 and $10^{\circ}$ standard observer. The washing fastness of dyeings was tested according to GB/T 3921-2008.

\section{Results and Discussion}

Effect of Softening Treatment on the Softness of Silk/PLA Fabric. The softness of textiles is the most important indicator which evaluates the quality of a softener. The effect of softening treatment on the softness of silk/PLA fabric is shown in Table 2. The RS-WR softener with fatty acid amide structure imparted the best softness to the fabric, whereas the CS-L softener with cationic fatty imidazoline structure provided the worst softness. The softness of the fabrics treated by the ALS-P, RS-C and RS-M softeners was between those treated by the CS-L and RS-WR softeners.

Table 2. Softness of the treated silk/PLA fabrics

\begin{tabular}{llllll}
\hline Softener & ALS-P & CS-L & RS-WR & RS-C & RS-M \\
\hline Fabric softness & 6.5 & 6.0 & 7.0 & 6.5 & 6.5 \\
\hline
\end{tabular}

Effect of Softening Treatment on the Hydrophilicity of Silk/PLA Fabric. The hydrophilicity of Silk/PLA fabric can be expressed in terms of the wetting time of a water droplet on the fabric surface, the contact angle of water on the fabric surface, the moisture regain defined as the percentage ratio of the weight of water to the oven dry weight of the fabric, and the capillary value expressed by the rising height of water along the fabric length orientation in the vertical wicking test. In the present work, the capillary effect of the fabric was used. Fig. 1 shows that the capillary values of the untreated fabric in the warp and weft orientations were 9.9 and $8.2 \mathrm{~cm}$, respectively. This reveals that the silk/PLA fabric has good hydrophilicity which is caused by the hydrophilic silk.

From Fig. 1, it is clear that all the softening treatments decreased the hydrophilicity of silk/PLA fabric, and the decrement extent of the hydrophilicity was dependent on the chemical nature of 
softeners. The ALS-P, CS-L and RS-WR softeners are amide ester, cationic fatty imidazoline and fatty acid amide derivatives, respectively; the fabrics treated by them showed poor hydrophilicity. However, the RS-C and RS-M softeners had little influence on the hydrophilicity of the fabric due to their polyether fatty acid ester component. Thus their applications can guarantee the inherent hydrophilicity of silk/PLA fabric.
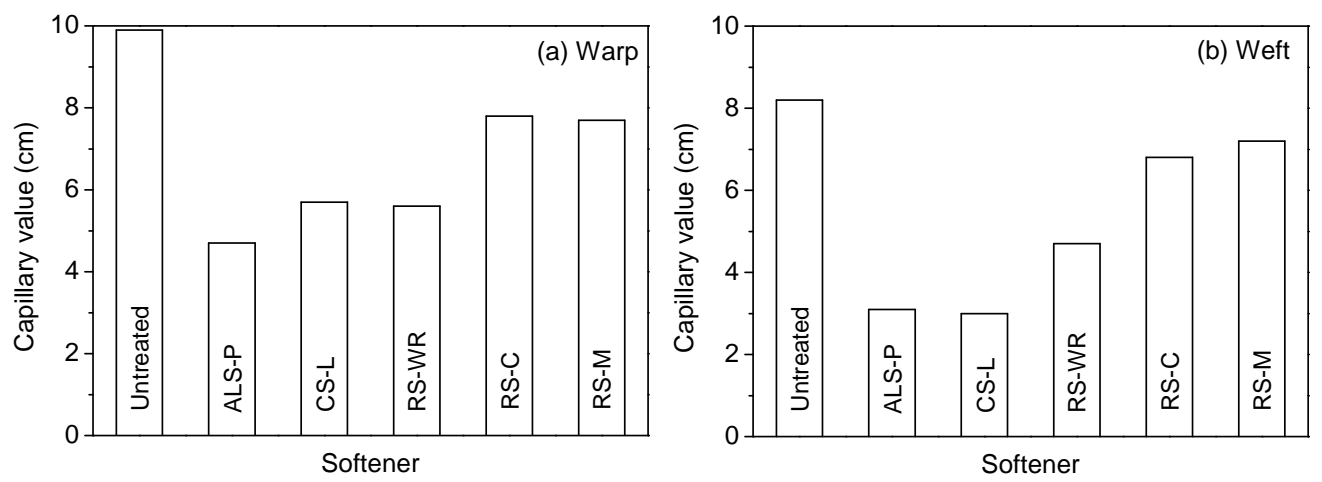

Fig. 1 Influence of softening treatment on the capillary effect of the treated silk/PLA fabrics

Effect of Softening Treatment on the Color Parameters and Fastness of the Dyed Silk/PLA Fabric. In the process of heat treatment, disperse dyes can migrate on and within the fibers and fabrics [5]. If a softener has affinity to dyes, it will accelerate the migration of dyes into the finish layer on the fiber surface. The migration of dyed can induce the color parameters and fastness of the dyed fabrics. Therefore, the effects of softening treatment on the color parameters and fastness of the dyed silk/PLA fabric were determined, and the related results are listed in Tables 3 and 4, respectively.

The used silk/PLA fabric has a satin weave, and its front side shows silk surface while its back side is PLA surface. When a disperse dye is applied to the dyeing of the fabric, the back side displays deeper shade than the front side because of the higher affinity of disperse dye to PLA fiber. This is proved by the fact that the back side of the dyed fabric without softening showed higher color strength $(\mathrm{K} / \mathrm{S})$, lower color lightness $\left(\mathrm{L}^{*}\right)$, and higher chroma $\left(\mathrm{C}^{*}\right)$ than the front side, as shown in Table 3. Softening treatment did not change the color hue of the dyed fabric according to the fluctuation of the $\mathrm{h}$ values in the order of magnitude. However, softening treatment changed the color depth of the dyed fabric.

Table 3. Color parameters of the dyed and softened silk/PLA fabrics

\begin{tabular}{llllll}
\hline Fabric side & Softener & K/S & $\mathrm{L}^{*}$ & $\mathrm{C}^{*}$ & $\mathrm{~h}$ \\
\hline Front side & No softener & 2.237 & 58.19 & 36.40 & 354.97 \\
& ALS-P & 2.176 & 59.09 & 36.57 & 356.43 \\
& CS-L & 1.982 & 60.28 & 35.91 & 355.83 \\
& RS-WR & 2.053 & 60.10 & 36.78 & 357.45 \\
& RS-M & 2.209 & 59.41 & 37.97 & 355.93 \\
Back side & No softener & 4.380 & 53.16 & 47.17 & 1.62 \\
& ALS-P & 3.848 & 55.12 & 46.19 & 1.22 \\
& CS-L & 3.825 & 55.31 & 46.38 & 1.14 \\
& RS-WR & 3.965 & 55.23 & 47.43 & 2.42 \\
& RS-M & 4.019 & 54.95 & 47.17 & 0.82 \\
\hline
\end{tabular}

The CS-L softener with cationic fatty imidazoline structure had the biggest influence on color depth, and the CS-L softened fabric showed lowest K/S, higher color lightness ( $\left.\mathrm{L}^{*}\right)$, and lowest $\mathrm{C}^{*}$. The RS-M softener which consists of polyether fatty acid ester and a small amount of fatty acid amide exerted the minimal impact on color depth, and there was the smallest difference of $\mathrm{K} / \mathrm{S}$, $\mathrm{L}^{*}$ and $\mathrm{C}^{*}$ 
between the unsoftened and softened fabrics was found. The ALS-P and RS-WR softeners had a certain extent of impact on color depth. The influence of softening treatment on color depth should depend on many factors such as the hydrophilicity and hydrophobicity of a softener, the affinity of a softener to disperse dye, the inherent color of a softener, the dependence of dye migration on a softener, the direction of dye migration (on fiber and fabric surface and between individual fibers). The RS-M softener imparted the best hydrophilicity to the fabric, and moreover had the minimal impact on color depth.

From Table 4, it is evident that softening treatment was able to affect the wash color fastness of the dyed fabric. But the fluctuation of wash fastness was in the half rating range. The RS-M softener was able to increase the fastness of the dyed fabric by half rating. The CS-L and RS-WR decreased the fastness of silk staining by half rating.

Table 4. Wash color fastness of the dyed and softened silk/PLA fabrics

\begin{tabular}{llll}
\hline Softener & $\begin{array}{l}\text { Color } \\
\text { change }\end{array}$ & Staining & \\
\cline { 3 - 4 } & $4-5$ & 4 & Polyester \\
\hline No softener & $4-5$ & 4 & $4-5$ \\
ALS-P & 5 & $3-4$ & $4-5$ \\
CS-L & $4-5$ & $3-4$ & $4-5$ \\
RS-WR & $4-5$ & $4-5$ & 5 \\
RS-M & 5 & &
\end{tabular}

\section{Conclusions}

The different softeners were applied to improve the handle of silk/PLA fabric. The effects of softening treatment on the handle, hydrophilicity, color parameters and color fastness of silk/PLA fabric were found to depend on their chemical structures. The softener having fatty acid amide structure imparted the best softness to the fabric. The polyether fatty acid ester softener had little influence on the hydrophilicity of the fabric as well as the color parameters and wash fastness of the dyed fabric.

\section{Acknowledgements}

This work was financially supported by Jiangsu Provincial Natural Science Foundation of China (BK2012197), Joint Innovation Fund of Jiangsu Province of China (BY2014059-04), Suzhou Science and Technology Support Program (SS201422), and the Priority Academic Program Development (PAPD) of Jiangsu Higher Education Institutions.

\section{References}

[1] J. Lunt and A.L. Shafer: J. Ind. Text. Vol. 29 (2000), p. 191

[2] G. Li, H. Liu, T. Li and J. Wang: Mater. Sci. Eng. C, Vol. 32 (2012), p. 627

[3] R.H. Yang and C.W. Kan: Fiber. Polym., Vol. 14 (2013), p. 1347

[4] B. Wahle and J. Falkowski: Rev. Prog. Color., Vol. 32 (2002), p. 118

[5] O. Avinc, M. Wilding, J. Bone, D. Phillips and D. Farrington: Color. Technol., Vol. 126 (2010), p. 353 